\title{
The role of oral health in gastrointestinal malignancies
}

\author{
Christian Madsen \\ Department of Dentistry, Hackensack Meridian Health, Jersey Shore University Medical Center, Neptune City, NJ, USA \\ Correspondence to: Christian Madsen, DMD. Department of Dentistry, Hackensack Meridian Health, Jersey Shore University Medical Center, \\ Neptune City, NJ 07753, USA. Email: Christian.Madsen@hackensackmeridian.org.
}

\begin{abstract}
Many studies have shown a connection between poor oral health and a number of systemic diseases. Recent studies have demonstrated an association between poor oral health and the oral microbiota to carcinogenesis. This review article will focus on studies that link oral dysbiosis, periodontal disease, and specific oral microorganisms to gastrointestinal malignancies. These studies provide some insight and understanding of the role of the oral microbiome in carcinogenesis. Understanding how the oral microbiota is related to carcinogenesis and gastrointestinal malignancies could lead to identifying biomarkers and provides future treatment modalities. More research in this area would be beneficial to the diagnosis and treatment of patients with gastrointestinal malignancies.
\end{abstract}

Keywords: Oral Health; periodontitis; gastrointestinal malignancies

Submitted Dec 19, 2019. Accepted for publication Jan 22, 2020.

doi: 10.21037/jgo.2020.02.03

View this article at: http://dx.doi.org/10.21037/jgo.2020.02.03

\section{Introduction}

The role of oral health and its association to colorectal cancer and other gastrointestinal malignancies has been the subject of many research articles and studies over the past several years. Many studies have focused on tooth loss, periodontal disease, chronic inflammation, oral microbiome, and their relationship to gastrointestinal cancer.

The oral microbiome has been related to a number of systemic diseases. These diseases include cardiovascular, pneumonia, heart disease, rheumatoid arthritis, colorectal cancer, esophageal cancer, pancreatic cancer, stroke and adverse pregnancy problems (1). Determining whether oral health status is a factor related to gastrointestinal could help in identifying and treating these malignancies.

A meta-analysis of colorectal cancer risk factors concluded that there is a significantly higher risk of colorectal cancer for both a diagnosis of inflammatory bowel disease and a history of colorectal cancer in a first-degree relative (2). There was also a significant risk associated with a high BMI, tobacco smoking and red meat consumption (2). Although this study is very inclusive, emerging studies have shown a possible link of oral health, periodontal disease, and the oral microbiome to gastrointestinal malignancies.
This paper will discuss the relationships between the oral microbiome, periodontitis and gastrointestinal malignancies. In addition, this paper will highlight systemic inflammation and the possible role of specific oral microorganisms in gastrointestinal cancers. Understanding the connection between oral health and gastrointestinal malignancies could lead to better management, diagnosis and treatment of these cancers.

\section{The oral microbiome}

The oral cavity is the gateway between the external environment and the gastrointestinal tract. The oral cavity contains over 700 microbiota species along with fungi, archaea, protozoa and viruses.1 The Human Oral Microbial Database collected 165 rRNA gene sequences. The databases includes 619 taxa in 13 phyla are follows: Actinobacteria, Bacteroidetes, Chlamydiae, Chloroflexi, Euryarchaeota, Firmicutes, Fusobacteria, Proteobacteria, Spirochaetes, SRI, Synergistetes, Tenericutes, and TM7 (3).

The oral microbiome plays an important role in the balance between health and disease. Every microbial player has its own role in the oral ecosystem regulation and in the symbiotic relationship between the oral microbiome and the 
host. Oral diseases are associated with a reduced microbial diversity and an oral dysbiosis. However, it is unclear whether disease is caused by loss of diversity or whether the specific niche in pathological tissue represents a very selective environment resulting in less diverse microbial ecology (4).

\section{Periodontal disease}

Periodontitis is an infection induced low-grade chronic inflammation that results in the loss of connective tissue attachment and bone support of teeth. The worldwide prevalence of periodontal disease ranges from 10-15\% (5). Risk factors such as poor oral hygiene, smoking, diabetes, medication, hereditary, age and stress are related to periodontal disease (6).

The microorganisms identified in the development of periodontal disease include Porphyromanos gingivalis, Actinobacillis Actinomycetemcomitans, Tannerella Forsythensis and Treponema denticola. The progression of periodontal disease is signaled by a change in the bacterial makeup in the dental biofilm from largely anerobic grampositive bacteria to a more pathogenic infectious state dominated by a composition of anerobic gram-negative organisms (7). Fusobacterium nucleatum is also a common gram-negative anaerobe associated with periodontitis (8).

Previous literature has shown that the systemic markers of inflammation associated with periodontal disease were increased as compared to unaffected controlled groups (9). C-reactive protein and interleukin- 6 were elevated in individuals with periodontal disease when compared to healthy individuals (10). Additionally, a study has shown that individuals that received periodontal therapy exhibited a significant decrease in serum $\mathrm{C}$-reactive protein and interleukin-6 (11). This shows that periodontitis may add to systemic inflammatory burden effective individuals (11).

Periodontitis and its resultant systemic inflammation has been associated with numerous systemic diseases. Studies have shown an association with cardiovascular disease, diabetes, adverse pregnancy outcomes, rheumatoid, arthritis, respiratory diseases, chronic kidney disease, impairment of cognitive function and cancers (6). Researchers have estimated that $15 \%$ of human tumors are a result of inflammation (12).

Studies have shown that the microbiota connected with periodontal disease can produce a systemic bacteremia (13). These oral bacteria can travel to extra-oral host cells and induce inflammation. This systemic inflammation has been associated with a number of systemic disease (13).
Since evidence supports an association between chronic inflammation and cancer, this suggests a link between chronic periodontal disease and cancer. Although the exact mechanism involved in carcinogenesis by oral and periodontal microorganisms are not known at this time, studies have demonstrated that inflammatory mediators produced as a result of periodontal disease could mediate carcinogenesis or these oral microorganisms can directly promote cellular transformation.

\section{Periodontal disease and its association with colorectal adenoma}

A recent study of 42.871 individuals at Kavgbuk Samsung Hospital in South Korea investigated whether there is an association between periodontitis and the risk of colorectal adenoma in asymptomatic healthy people (14). The results of the study concluded that periodontitis when compared to other risk factors, the odds ratio (OR) of periodontitis was similar to that of smoking 10-20 pack of cigarettes per year or a moderate amount of alcohol intake (14). This study suggests that periodontitis and poor oral health may be at risk factor for colorectal adenoma. The study concluded that further studies of the association between oral health and gastrointestinal neoplasm may help to identify and prevent gastrointestinal malignancy (14).

\section{Periodontal disease and its association with precancerous lesions of gastric cancer}

A study hypothesized that individuals with poor periodontal conditions would have an increased periodontal pathogen burden and changes in the microbial diversity, leading to an increased risk of precancerous lesions of gastric cancer (PLGC) (15). The results of this study that compared the control group patients with PLGC experience a high level of bleeding on probing, high levels of T. Denticola and A. Actinomycetemcomitans (periodontal disease associated microorganisms) and less bacterial diversity in saliva and dental plaque (15). This study concluded that the periodontal pathogenic burdens and decreased bacterial diversity in the oral cavity are important factors contributions to a potential increased risk of developing precancerous lesions of gastric cancer (15).

\section{Periodontitis and $\boldsymbol{H}$. pylori infection}

A study completed by Zheng and Zhou discovered a correlation 
between periodontal health status and $H$. pylori infection (16). Individuals in this study with moderate to severe periodontal disease showed a significantly higher urease $\mathrm{C}$ gene and cag A gene of $H$. pylori than these of the control (16). This study concluded that periodontal health status of individuals with periodontitis correlated with $\mathrm{H}$. pylori infection in the stomach (16).

In another study the prevalence of $H$. pylori in the oral cavity and stomach were compared to determine if a relationship between oral $H$. pylori and gastric $H$. pylori exists (17). In this study, oral $H$. pylori was present in the dental plaque in $263(59.4 \%)$ of the 443 individuals and gastric H. pylori in the stomach of $273(61.6 \%)$ individuals (17). The prevalence of gastric infection was significantly higher in those individuals with a positive test for $H$. pylori in their dental plaque than in the individuals with no $H$. pylori infection in dental plaque (17). This study concluded the presence of $H$. pylori in the oral cavity might be a source for $H$. pylori infection in the stomach (17).

A recent study by Yuksel Sert et al. studied the effect of periodontal treatment and oral hygiene on the eradication of gastric $H$. pylorii (18). In this study, individuals with gastric $H$. pylori infection were placed into two groups (18). One group received triple-therapy regime and the other received triple-therapy regime and periodontal treatment (18). The triple-therapy regime consisted of a 10-day course of a Proton-Pump-Inhibitor (PPI) with amoxicillin ( $2 \times 1$ gram daily) and clarithromycin $(2 \times 500 \mathrm{mg}$ daily) $(18)$. Periodontal therapy consisted of scaling, root planning, and oral hygiene instructions (18). The triple-therapy regime plus periodontal treatment resulted in a $64.7 \%$ eradication rate, while the triple therapy regime alone resulted in a $51.1 \%$ (18). Additionally, it was noted oral hygiene and plaque control improved the $H$. pylori eradication rate (18). This study provides evidence of the importance of periodontal therapy and oral hygiene on achieving a satisfactory elimination of gastric $H$. pylori (18).

\section{Oral microbiome and gastrointestinal cancer}

Cancer is a multifaceted disease influenced by genetic factors and the environment. The mechanisms by which and the role the microbiome influence carcinogenesis are not fully understood. Over the past several years, research has linked oral microorganisms to gastrointestinal cancer. Studies are providing more evidence that the microbiome has more a role in gastrointestinal malignancies than previously thought. The possible mechanisms that the oral microbiota induced carcinogenesis are the induction of chronic inflammation, local metabolism of carcinogens, interferences of cell cycles and immune regulation (19). The association of $H$. pylori to gastric cancer may be reason for increase in research in the area (20).

Several studies have explained the association between the oral microbiome and gastrointestinal cancers. Ahn et al. examined whether periodontal disease is associated with orodigestive cancer mortality (21). The study addressed directly the microbe-cancer association by investigating the relationship of serum antibody levels for $P$. gingivalis in relation to orodigestive cancer mortality (21). The results showed that orodigestive cancer was increased in individuals with periodontal disease (21). There was also evidence that orodigestive cancer mortality increased with the severity of periodontal disease (21). Individuals with periodontal disease had excess mortality due to colorectal and pancreatic cancer (21). Participants with high levels of serum antibody to $P$. gingivalis also tended to have excess orodigestive cancer mortality overall (21). This study showed an increasing risk for orodigestive cancer mortality in relation to increasing severity of periodontal disease in relation to serum $P$. gingivalis IgG, a biomarker for exposure to this periodontitis related pathogen (21). Although the research sample size was small it appears that there is an association between pancreatic and colorectal cancer and individuals with periodontal disease (21).

A study completed in 2012 by Michaud et al. measured the plasma antibodies to 25 oral bacteria in 405 pancreatic cancer patients and 416 matched controls (22). This study showed that patients with high antibodies against $P$. gingivalis (ATTC Strain 53978) a pathogenic periodontal bacteria had a two fold higher risk of pancreatic cancer than individuals with lower levels of these antibodies (22). This may indicate a possible association between periodontal disease and pancreatic cancer (22). More research is needed to determine if oral bacteria have direct effects on pancreatic cancer pathogenesis or can be a potential biomarker (22).

In another study, Castellarin et al. evaluated frozen sections of 11 matched pairs of colorectal carcinoma and adjacent normal tissue specimens (23). The only alignments obtained from the results that were remarkable was a higher rate of a gram negative microorganisms, Fusobacterium nucleatum (ATTC Strain 25586), associated with periodontal disease in the tumor versus the control tissue (23). In 9 of the 11 tissue specimens showed at least a twofold higher read counts of $F$. nucleatum in the tumor tissue 
relative to the corresponding control tissue (23).

Ito et al. evaluated the presence of Fusobacterium nucleatum in 465 premalignant lesions and 511 colorectal cancer lesions (24). The study concluded that $F$. nucleatum was identified in premalignant colorectal cancer lesions but were more frequent in $\mathrm{CpG}$ island methylator phenotype (CIMP) high lesions (24). F. nucleatum was significantly higher $(56 \%)$ in colorectal cancer lesions than in many premalignant lesions (24). F. nucleatum increased accordingly to the increasing histological grade of the lesions (24). This study suggests that $F$. nucleatum may contribute to the progression of colorectal cancer (24).

Rubinstein et al. studied the relationship between $F$. nucleatum and the promotion of colorectal carcinogenesis by modulating $\mathrm{E}$-cadherin/ $\beta$-catenin signaling via its Fusobacterium adhesion A (FadA) (25). The study demonstrated the F. nucleatum binds to both normal and cancerous epithelial cells via FadA binding to epithelial E-cadherin activating $\beta$-catenin signaling leading to an increase in the expression of transcription factors, oncogenes, Wnt genes, inflammatory genes and growth stimulation of CRC cells (25). Colon tissue from patients with adenomas and adenocarcinomas had FadA levels $>10-100$ times higher when compared to normal individuals (25). The FadA binding site on E-cadherin has been mapped to an 11 amino acid region (25). A synthetic peptide corresponding to this region prevents $F$. nucleatum from binding and invasion of colorectal cancer cells (25). This abolishes induced CRC cell growth, oncogenic and inflammatory responses, and gene expression (25). This study makes it evident that $F$. nucleatum contributes to colorectal cancer and identifies FadA as a potential diagnostic and therapeutic target for CRC (25).

These studies all show how the oral microbiota may play a role in gastrointestinal cancers. Further studies are required to investigate the underlying mechanisms of how the oral microbiota induce carcinogenesis and gastrointestinal malignancies. These future studies will be beneficial to the prevention, diagnosis, and treatment of these cancers.

\section{Conclusions}

There is increasing evidence for an association between poor oral hygiene, periodontitis, the oral microbiome, and gastrointestinal cancer. Studies have shown that treatment and reduction of periodontal disease has resulted in improved systemic disease.
Healthcare providers should be familiar with the oralhealth/systemic-health link and the impact of oral-health on a patients' overall health. Also, healthcare providers should educate their patients on the importance of oral healthcare. Based on the findings and information from the literature reviewed, it is important for those patients diagnosed with a premalignant or malignant gastrointestinal tumor to be referred to a dentist for a comprehensive oral examination. Those patients diagnosed with periodontal disease should receive the appropriate periodontal treatment and to continue with periodontal maintenance and good oral hygiene.

Additional studies are needed to focus on the association between oral health and gastrointestinal cancers. This will help in the possible prevention, diagnosis and treatment of gastrointestinal cancers. The emerging field of molecular pathological epidemiology (MPE) may provide the clues about risk factors, mechanisms of disease and biomarkers. This knowledge may help in understanding the link between oral health and gastrointestinal malignancies. This in turn may lead to strategies for disease prevention and treatment.

\section{Acknowledgments}

Funding: None.

\section{Footnote}

Provenance and Peer Review: This article was commissioned by the Guest Editors (John F. Gibbs and Quyen D. Chu) for the series "Global GI Malignancies" published in Fournal of Gastrointestinal Oncology. The article has undergone external peer review.

Conflicts of Interest: The author has completed the ICMJE uniform disclosure form (available at http://dx.doi. org/10.21037/jgo.2020.02.03). The series "Global GI Malignancies" was commissioned by the editorial office without any funding or sponsorship. The author has no other conflicts of interest to declare.

Ethical Statement: The author is accountable for all aspects of the work in ensuring that questions related to the accuracy or integrity of any part of the work are appropriately investigated and resolved.

Open Access Statement: This is an Open Access article distributed in accordance with the Creative Commons 
Attribution-NonCommercial-NoDerivs 4.0 International License (CC BY-NC-ND 4.0), which permits the noncommercial replication and distribution of the article with the strict proviso that no changes or edits are made and the original work is properly cited (including links to both the formal publication through the relevant DOI and the license). See: https://creativecommons.org/licenses/by-nc-nd/4.0/.

\section{References}

1. Jia G, Zhi A, Lai PFH, et al. The oral microbiota a mechanistic role for systemic diseases. Br Dent J 2018;224:447-55.

2. Johnson CM, Wei C, Ensor JE, et al. Meta-analyses of colorectal cancer risk factors. Cancer Causes Control 2013;24:1207-22.

3. Dewhirst FE, Chen T, Izard J, et al. The human oral microbiome. J Bacteriol 2010;192:5002-17.

4. Krom BP, Kidwai S, Ten Cate JM. Candida and other fungal species: forgotten players of healthy oral microbiota. J Dent Res 2014;93:445-51.

5. Kassebaum NJ, Bernabé E, Dahiya M, et al. Global Burden of Severe Tooth Loss: A Systematic Review and Meta-analysis. J Dent Res 2014;93:20S-8S.

6. Nazir MA. Prevalence of periodontal disease, its association with systemic diseases and prevention. Int $\mathrm{J}$ Health Sci (Qassim) 2017;11:72-80.

7. Moutsopoulos NM, Madianos PN. Low-grade inflammation in chronic infectious diseases: paradigm of periodontal infections. Ann N Y Acad Sci 2006;1088:251-64.

8. Yang NY, Zhang Q, Li JL, et al. Progression of periodontal inflammation in adolescents is associated with increased number of Porphyromonas gingivalis, Prevotella intermedia, Tannerella forsythensis, and Fusobacterium nucleatum. Int J Paediatr Dent 2014;24:226-33.

9. Loos BG. Systemic markers of inflammation in periodontitis. J Periodontol 2005;76:2106-15.

10. Sun XJ, Meng HX, Shi D, et al. Elevation of C-reactive protein and interleukin- 6 in plasma of patients with aggressive periodontitis. J Periodontal Res 2009;44:311-6.

11. D'Aiuto F, Parkar M, Andreou G, et al. Periodontitis and systemic inflammation: control of the local infection is associated with a reduction in serum inflammatory markers. J Dent Res 2004;83:156-60.

12. Heikkilä P, But A, Sorsa T, Haukka J. Periodontitis and cancer mortality: Register-based cohort study of 68,273 adults in 10-year follow-up. Int J Cancer 2018;142:2244-53.

13. Han YW, Wang X. Mobile microbiome: oral bacteria in extra-oral infections and inflammation. J Dent Res 2013;92:485-91.

14. Lee D, Jung KU, Kim HO, et al. Association between oral health and colorectal adenoma in a screening population. Medicine 2018;97:e12244.

15. Sun J, Zhou M, Salazar CR, et al. Chronic Periodontal Disease, Periodontal Pathogen Colonization, and Increased Risk of Precancerous Gastric Lesions. J Periodontol 2017;88:1124-34.

16. Zheng $\mathrm{P}$, Zhou $\mathrm{W}$. Relation between periodontitis and helicobacter pylori infection. Int J Clin Exp Med 2015;8:16741-4.

17. Liu Y, Yue H, Li A, et al. An epidemiologic study on the correlation between oral Helicobacter pylori and gastric $\mathrm{H}$. pylori. Curr Microbiol 2009;58:449-53.

18. Yuksel Sert S, Ozturk A, Bektas A, et al. Periodontal treatment is more effective in gastric Helicobacter pylori eradication in those patients who maintain good oral hygiene. Int Dent J 2019;69:392-9.

19. Zhang $Y$, Niu Q, Fan W, et al. Oral microbiota and gastrointestinal cancer. Onco Targets Ther 2019;12:4721-8.

20. Parkin DM. The global health burden of infectionassociated cancers in the year 2002. Int J Cancer 2006;118:3030-44.

21. Ahn J, Segers S, Hayes RB. Periodontal disease, Porphyromonas gingivalis serum antibody levels and orodigestive cancer mortality. Carcinogenesis 2012;33:1055-8.

22. Michaud DS, Izard J, Wilhelm-Benartzi CS, et al. Plasma antibodies to oral bacteria and risk of pancreatic cancer in a large European prospective cohort study. Gut 2013;62:1764-70.

23. Castellarin M, Warren RL, Freeman JD, et al. Fusobacterium nucleatum infection is prevalent in human colorectal carcinoma. Genome Res 2012;22:299-306.

24. Ito M, Kanno S, Nosho K, et al. Association of Fusobacterium nucleatum with clinical and molecular features in colorectal serrated pathway. Int J Cancer 2015;137:1258-68.

25. Rubinstein MR, Wang X, Liu W, Hao Y, Cai G, Han YW. Fusobacterium nucleatum promotes colorectal carcinogenesis by modulating $\mathrm{E}$-cadherin/ $\beta$-catenin signaling via its FadA adhesin. Cell Host Microbe 2013;14:195-206.

Cite this article as: Madsen C. The role of oral health in gastrointestinal malignancies. J Gastrointest Oncol 2021;12(Suppl 2):S311-S315. doi: 10.21037/jgo.2020.02.03 